Ebisu

Études japonaises

Études japonaises

49 | printemps-été 2013

De chose en fait : la question du milieu

\title{
La raison-cœur des co-suscitations paysagères : les fluctuations du paysage entre corps, lieu et langage
}

The Reason-Sentiment of Landscape Co-susceptibility: Landscape Fluctuations between Body, Place and Language

縁起の情理による風景論一身体、場所、言語のあいだに浮遊する風 景一

\section{Yoshio Nakamura}

Traducteur : Augustin Berque

\section{(2) OpenEdition}

\section{Journals}

Édition électronique

URL : http://journals.openedition.org/ebisu/766

DOI : $10.4000 /$ ebisu.766

ISSN : 2189-1893

Éditeur :

Institut français de recherche sur le Japon (UMIFRE 19 MAEE-CNRS), Maison franco-japonaise

\section{Édition imprimée}

Date de publication : 1 avril 2013

Pagination : 73-90

ISSN : 1340-3656

\section{Référence électronique}

Yoshio Nakamura, «La raison-cœur des co-suscitations paysagères : les fluctuations du paysage entre corps, lieu et langage », Ebisu [En ligne], 49 | printemps-été 2013, mis en ligne le 04 avril 2014, consulté le 30 avril 2019. URL : http://journals.openedition.org/ebisu/766 ; DOI : 10.4000/ebisu.766 


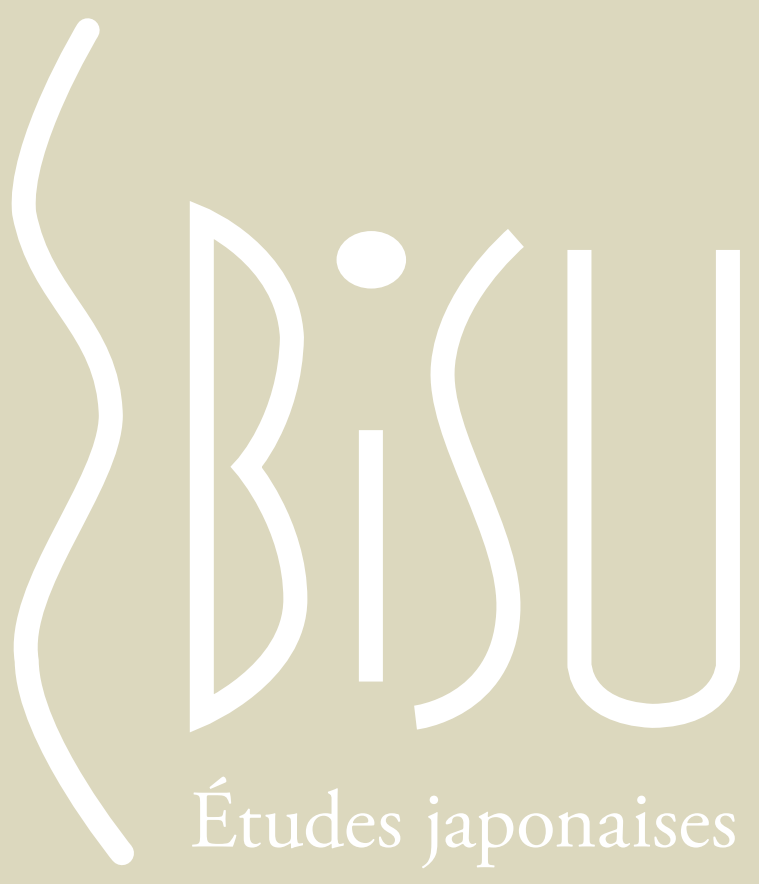

Dossier

De chose en fait : la question du milieu

Articles issus du colloque de Shin-Hirayu

Coordonné par Augustin BERQue

Varia | Nicolas Baumert - Peut-il exister des terroirs du saké ?

Conférences de la MFJ | François Macé - Le Kojiki, une Énéide longtemps oubliée ? | Patrick Beillevaire - Présences françaises à Okinawa : de Forcade (1844-1846) à Haguenauer (1930)

Livres à lire

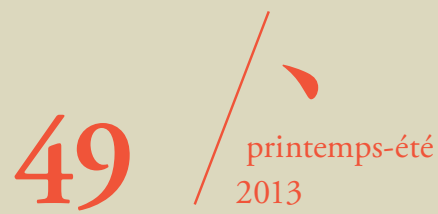




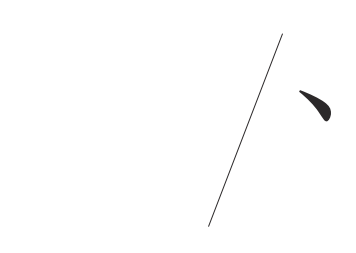

\section{Dossier}

\section{De chose en fait : la question du milieu}

Mono kara koto e to füdo no mondai

物から事へと風土の問題

Articles issus $d u$

colloque de Shin-Hirayu

Dossier coordonné

par Augustin Berque 


\section{La raison-cœur des co-suscitations paysagères Les fluctuations du paysage entre corps, lieu et langage}

NAKAMURA Yoshio 中村良夫*

\section{Co-suscitation et paysage vécu}

Dans un quatrain en heptasyllabes ${ }^{1}$ de Xuē Tāo 薛濤 (768-831 ?), poétesse de l'époque Tang, on peut lire ce vers :

春教風景駐仙霞

Chün jiào fêngjīng zhù xiān xiá

Le printemps fait s'arrêter un nuage féérique dans le paysage

(jp. Haru wa fükei o shite senka o todomarashime)

Sans doute le mot fükei (en chinois féngjĭng 風景, paysage) était-il connu également au Japon, à l'époque Heian, par les lettrés et la noblesse, mais

* Professeur honoraire à l'Institut de technologie de Tokyo (東京工業大学).

1. N.D.T. Voici, trouvée sur Internet (http://www.haoshici.com/Xuetao4809.html), l'intégralité de ce quatrain, intitulé "Le val des pommiers à bouquets " (海棠溪 Hăitáng $x \bar{i}$ ) [le pommier à bouquets est Malus spectabilis (Ait.) Borkh.] : 春教風景駐仙霞 / 水面魚 身總帶花 / 人世不思靈卉異 / 竟將紅縝染輕紗 chün jiào fêng jing zhù xiān xiá / shui miàn yú shèn zǒng dài huā / rén shì bù sī líng hui yì / jìng jiāng hóng xié răn qīng shā; ce dont on peut risquer la traduction suivante : En nuage de fées le printemps fait s'arrêter le paysage / Le corps des poissons est tout fleuri par la surface de l'eau / Herbes étranges de l'âme que le monde ignore / La gaze légère en vient à se moirer de rouge (le traducteur remercie Chang Yunting, Fang Xiaoling et Zhang Chunyan pour leur aide). 
il ne s'est fixé dans la langue japonaise que beaucoup plus tard, semble-t-il vers la fin du shogunat ou sous Meiji. Quant à l'autre mot aujourd'hui courant pour " paysage ", keikan 景観, l'on suppute, selon Tsujimura Tarō 辻村太郎 (1937), que ce sinolexe nippon (wasei kango 和製漢語) fut employé pour la première fois par le botaniste Miyoshi Manabu 三好学 pour traduire l'allemand Landschaft dans son ouvrage de 1905, Paysages végétaux du Japon (Nihon shokubutsu keikan 日本植物景観).

La distinction entre les deux termes n'est pas toujours claire, mais keikan étant à l'origine un terme scientifique, on doit penser qu'il représente un objet qui s'oppose à un sujet qui l'observe. Alors, seule est en scène l'image de l'objet, et le regard concentré dans le point de vue du sujet se tient en retrait, caché hors du plan de l'image.

En revanche, le poème de Xuē Tão, qui chante le paysage printanier d'une vallée plutôt que le contour d'une forme objective, a pour thème le nuage floral indéfini dont les rosacées couvrent la terre. On entend de vive voix la poétesse, dont le corps, engagé dans la scène, y plante ses mots comme une broderie sur la terre en fleurs. Dans cette alliance du sentiment et de la scène (keijō ichimi 景情一味, Bashō 芭蕉), la description manifeste également l'autoréférence de l'auteur, qu'exclut au contraire le paysage keikan objectif.

Le paysage fükei apparaît dans un entre-deux, où interagissent les circonstances locales, qui refusent d'être un en-soi substantiel, et le corps qui y est engagé. Pour saisir ce caractère non platonicien, nous proposons d'employer la logique du Grand Véhicule, pour lequel les phénomènes sont vides de nature propre et se suscitent mutuellement, ce qui est la co-suscitation (engi 縁起 : pratītya samutpāda $)^{2}$.

2. Keikan 景観 et fükei 風景. Les deux termes ne sont jamais définis précisément. D'après la discussion précédente, l'auteur propose ses définitions comme suit. Keikan est l'image visuelle de l'environnement comme un objet indépendant du sujet, tandis que fükei n'est pas détaché du sujet, dont le corps avec tous ses sens est intégré dans cette expérience phénoménologique. D’autre part, le langage ou la connaissance s'engagent également dans le fükei en termes d'articulation sémiologique. Fükei se caractérise par cette ambiguïté et cette ambivalence ontologiques. C'est la manifestation synthétique, symbolique, poétique et donc suprême du füdo (milieu humain). 
Yamanouchi Tokuryū (1974) explique ainsi l'engi :

1. Étant vides de nature propre, les phénomènes n'adviennent point par causalité (kara から from), mais en vertu de (yotte よって by) leur relation de co-suscitation.

2. La causalité (inga 因果), qui se noue dans la nécessité du logos, est un rapport d'être (sonzai no kankei 存在の関係), tandis que la co-suscitation, qui procède du senti rationnel (jōri 情理), est un rapport de vide ( $k \bar{u} n o$ kankei 空の関係).

3. L'enchaînement nécessaire de la cause et de l'effet se déroule temporellement et unilatéralement, tandis que la co-suscitation est mutuelle et spatiale.

4. Le vide n'a en lui-même pas de nature propre substantielle, mais il noue des liens avec d'autres vides, engendrant ainsi les rapports de cosuscitation, et se contentant d'arrimer les choses par calage mutuel (eji 依止).

Selon Yamanouchi, par l'effet de ce calage, les choses constituent des domaines (Yamanouchi 1974 : 130). Ces domaines, comme les champs électromagnétiques ou gravifiques, peuvent être comparés à des aires de puissance latente, invisible mais s'exerçant sur les choses environnantes. Entre parents et enfants, époux et épouse, maître et disciple, etc., les humains vivent dans de tels champs, tendus de ces liens d'en 縁 appelés seken 世間 (monde, i.e. les gens). On pourrait dire que ce qui advient ainsi par co-suscitation est une phénoménologie du champ ( $b a$ no genshöron 場 の現象論).

À la différence des choses (mono モノ), qui sont connues par le dehors, découpées par un contour, et se dressent comme objets face au sujet, un champ ( $b a$ 場) n'est pas un objet. C'est un état émanant de l'énergie spirituelle du corps (shintai no seiki ga haru jōkyo 身体の精気が張る状況), un champ corporel (shintaiba 身体場), perçu avant tout par les sens du corps. À la différence de la vue, qui est propice à l'objectification, cet état s'accompagne de l'autoréférence du corps lui-même. Le terme « champ » s'applique ici comme en physique, où l'on parle de champ gravifique ou de champ électromagnétique. Ce n'est ni un espace vide ni un objet, mais un espace où se propage un effet ou un fait dont la génération est attribuée au corps humain vivant, très proche de la terre, ce qui produit une image très déformée de celle-ci, pleine de niches. Habituellement, de telles images des champs 
terrestres sont appelées « lieux » (basho 場所). Elles sont structurées par les traces vécues des corps, incarnées par des affordances (Gibson 1979) et illustrées par des cartes cognitives (Lynch 1963). Le champ est donc la base primitive des lieux, remplie de vie, tendue de naturalité et de corporéité.

Ne pourrait-on pas dire qu'un paysage (fūkei 風景) nous apparaît dans un rapport de co-suscitation (engi kankei 縁起関係) noué entre notre corps et un lieu, perçu comme une matrice ? Si la disposition du corps dans le lieu change, alors se composent de nouveaux rapports de co-suscitation. Le lieu et le corps, médiés par le paysage, sont fermement enchaînés par ces rapports co-suscitatifs.

C'est à peu près en ces termes que l'on comprendra que le paysage émerge en vertu d'une logique de la co-suscitation (engi no ronri 縁起の論 理). Pour en apprécier les divers aspects, voyons d'abord comment le corps se réfère à lui-même par son engagement dans le paysage.

\section{Le soi dans le paysage}

\subsection{Le champ des gestes potentiels du corps}

Dans la figure 1, tirée de l'Edo meisho zue 江戸名所図会 (Les hauts lieux d'Edo), on voit un personnage qui, la main en visière, contemple la mer au loin. C'est un point d'appel (tenkei 点景), qui invite le moi dans ce lieu où il ne figure pas. Le procédé du point d'appel est propre aux peintures de genre (füzokuga 風俗画) et aux peintures de lettrés (bunjinga 文人画). Il engage le moi dans cet espace, au moyen d'un « moi substitut » (dairi jiga 代理自我).

Contrairement à celui qu'enferment dans leur cadre les photographies ou les tableaux, le paysage grandeur nature n'est pas une image visuelle, objectivée et coupée du soi. Le champ visuel, dans le paysage qu'on a devant soi, est au contraire entrelacé de projections du sens tactile des bras, des pieds, des mains. Par exemple, un chemin de campagne nous invite à y marcher, l'eau d'un ruisseau ombragé nous semble fraîche, le gazon nous propose le plaisir de s'y asseoir. Le paysage (keikan 景観) qu'évide et objectifie le regard est en réalité empreint de notre corporéité par le toucher, l'ouïe, le goût et l'odorat; il en émane une énergie spirituelle.

En somme, le paysage (fükei 風景) n'est pas un moule objectivé par le regard, comme dans les tableaux ou les photographies; il porte la marque de nos cinq sens, qui en font un champ de vie (seikatsu no ba 生活の場) à 
habiter, occuper, utiliser. Dans la mesure où l'impression que nous avons d'un paysage porte ainsi la marque des sens de notre corps, ce n'est pas un objet séparé de nous-mêmes : il prolonge nos sens, et l'on peut dire en ce sens qu'il est autoréférentiel. Cette corporéité du paysage, c'est ce que Gibson a appelé affordance, y distinguant possiblement des affordances positives, où il y aurait projection active de nos gestes, et des affordances passives, où c'est l'ambiance qui nous envelopperait.

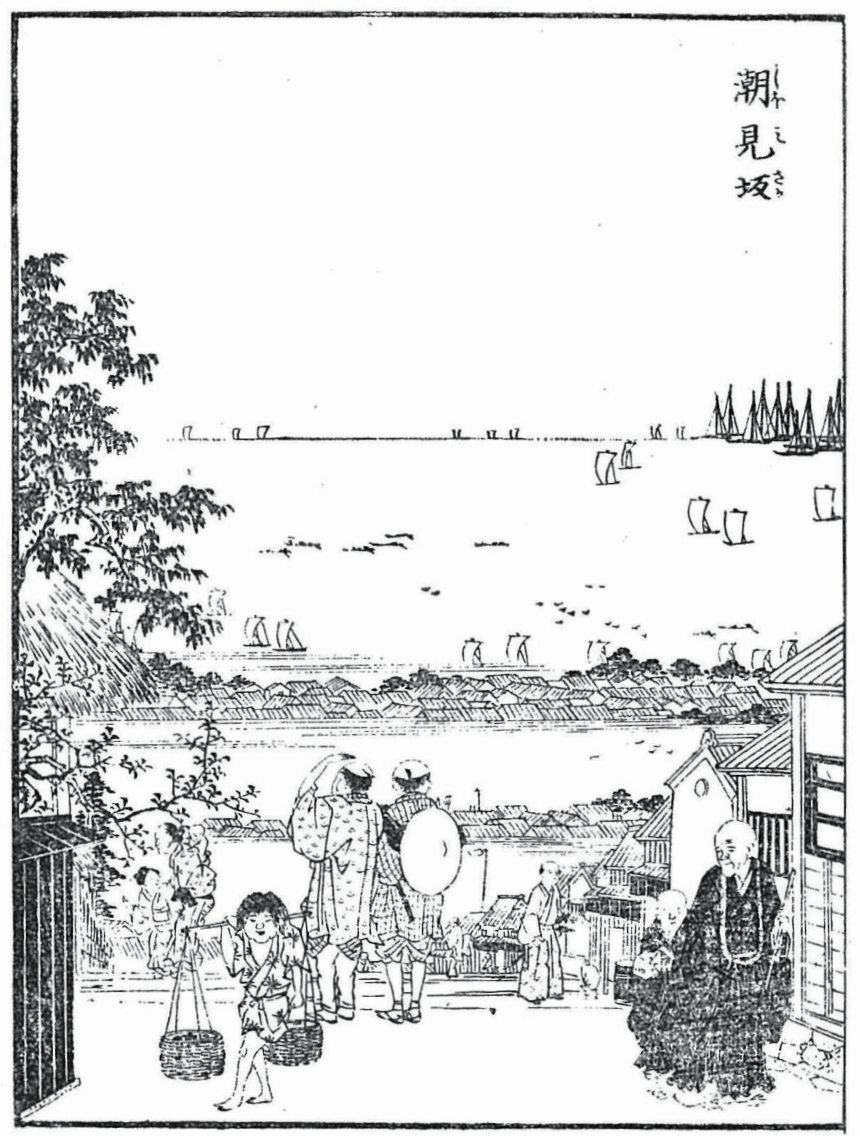

Fig. 1 Shiomizaka 潮見坂 (Edo meisho zue) 
Le monde sensible est un champ qui se manifeste gravé d'images du comportement potentiel de notre corps. Les points d'appel qui le composent, maisons, arbres, chemins, ponts, etc., ne sont pas des choses (mono モノ), mais agissent comme des signes médiateurs (shokubaiteki na kigō 触媒的な記号) qui déclenchent les gestes de notre corps. Ainsi, par un engagement du corps sans cause initiale, l'espace objectif devient un champ plein d'énergie spirituelle, parcouru de liens avec le corps. Cet espace excité par le corps, on peut l'appeler « champ corporel " (shintaiba 身体場). Comme les liens qui le tissent sont mutuels, ils incarnent aussi la façon de vivre du moi dans l'utilisabilité, l'habitabilité du paysage.

Significativement, cette expression sémiotique du champ comme trace corporelle est probablement analogue à ce qui, dans la terminologie d'Uexküll (1934), est le Merkbild (image-perception : ce que le vivant perçoit subjectivement), conditionné par le Wirkbild (image-action). Quoi qu'il en soit, ce rapport entre lieu et corps n'est pas déterminé causalement ; ne serait-ce donc pas une sensation arbitraire et créatrice, due à la co-suscitation ? Nous allons maintenant éclairer ce second aspect du rapport entre corps et lieu.

\subsection{Le soi à la lumière du lieu}

Regardons de nouveau la figure susdite. Dans cette image, un personnage tourne le dos au paysage marin. Il ne regarde pas la vue, on dirait au contraire qu'il marche en projetant le paysage environnant sur l'écran de son corps, et qu'il vit de la lumière du lieu.

Il arrive, indubitablement, que dans des lieux où l'on trouve des montagnes sacrées, révérées depuis des temps immémoriaux comme le mont Fuji, ou quand on marche dans des rues anciennes, l'on se sente bizarrement regardé par derrière. Dans ces moments-là, ce n'est pas moi qui regarde le paysage ; au contraire, ma conscience d'observateur recule, c'est quelqu'un d'autre qui me fixe de son regard, de sa posture, de sa silhouette. Comme l'écrit le Trésor de l'Cil de la Vraie Loi (Shōbōgenzō 正法眼蔵), c'est un "soi réalisé par tous les phénomènes " (banshō ni shöserareru jiko 万象 に証せられる自己) (Dōgen 1990 : 54), pourrait-on dire, qui s'éveille dans un état de passivité ; un soi que l'on pourrait appeler un "soi sans forme " (musō no jiko 無想の自己). 
Dans le cas des affordances, un certain répondant s'établit entre la vision de l'espace et les gestes potentiels du sujet. Il y subsiste donc quelque rapport de causalité. En revanche, quand le corps " vit de la lumière du lieu ", c'est dans la rencontre acausale du lieu et du corps, c'est-à-dire dans un rapport de co-suscitation, qu'apparaît le paysage.

\section{Co-suscitation et $m a$}

Le japonais appelle ma 間 la marge (yohaku 余白) temporelle et spatiale qui s'étend " entre » (aida あいだ) les choses et les choses, les choses et les humains, la terre et les humains; la liaison entre ces termes formant un champ empreint d'énergie spirituelle. Cela ne désigne pas un rapport de causalité entre des substances, mais une rencontre contingente entre ces termes, laissant flotter entre eux un je-ne-sais-quoi d'immatériel (nioitatsu $k \bar{u} s \bar{o}$ no kehai 匂い立つ空相の気配).

\subsection{Le ma entre terre et corps}

Revenons sur le phénomène signalé plus haut, où nous allons examiner deux sortes de ma qui émanent de l'entrelien (aida) d'un lieu terrestre et du corps humain.

La première sorte de ces ma est la hauteur du point de vue que l'on a sur la terre. À mesure que celui-ci s'abaisse, augmente la distorsion perspective de l'image terrestre. Les ondulations continues de la terre en viennent à se segmenter, les montagnes, les arbres, les maisons, etc., se particularisent, acquièrent un contour propre, laissant apparaître des silhouettes et des postures pleines d'expression. Toutefois, ces formes ne sont pas fermées comme les figures du gestaltisme. Certes, les montagnes dessinent sur le ciel une figure, mais leur piémont, libéré du joug de cette figure, se fond dans ce champ qu'est la terre. On a là une figure incomplète et ambivalente, inexprimable par la binarité du rapport figure/fond.

En même temps apparaissent à coup sûr, dans les défilements, les plis, les niches de ces images-perceptions nées de la distorsion perspective, toutes sortes de prises (tegakari 手がかり) et d'habitabilités (sumigokochi 住み心地) positives, emplies comme on l'a vu plus haut de gestes potentiels pour notre 
corps (se rapprocher, se mouvoir, tourner, traverser, toucher, se défendre, se terrer, observer...).

La deuxième sorte de ma est celle entre montagne et humain en direction horizontale.

Par exemple, la silhouette d'une montagne vue de loin disparaît quand on s'en rapproche, laissant place aux variations de la forêt qui couvre ses flancs. Si l'on se rapproche encore, on apercevra sans doute une scènerie composée de toits et de haies. Cela dénote une complexité générative, le paysage fonctionnant comme un emboîtement, un oignon que l'on pèle à mesure. La montagne et moi ne sommes pas dans le rapport d'opposition de deux individualités obstinées, mais de deux individualités flexibles qui, tout en nouant un lien répondant à la prise d'un certain $m a$, ne cessent de renouveler la visibilité du paysage (fükei no miegakari 風景の見えがか $り)$, tandis que sous ce regard s'engendre un soi tout frais. Pour reprendre l'expression de Yamanouchi, la montagne et moi ne sommes pas relatifs (sōtai 相対) l'un à l'autre, mais en coattente (sōdai 相待) l'un de l'autre, nous engendrant ainsi mutuellement. Simultanément à cette cosmophanie (atarashii sekai no kengen 新しい世界の顕現), s'engendre un soi défini par ce regard en devenir. Telle est sans doute la co-suscitativité du paysage (fükei no engisei 風景の縁起性). On peut dire qu'il naît dans la co-suscitation qu'est le $m a$.

Du fait de la coattentivité (sōdaisei 相待性) du corps et du lieu, les divers aspects du paysage peuvent changer complètement. Sur ce dernier point, l'on se reportera à la minutieuse étude de Baridon (1998).

\subsection{Les bordures (fuchi 縁)}

L'engawa 縁側 ou en 縁 (plateforme bordière dans la maison japonaise traditionnelle) est, dans l'architecture japonaise, la forme la plus riche en nuances. C'est par principe un ma indécis entre le dedans et le dehors, non point clos de fusuma 襖 (cloison coulissante opaque entre deux pièces) et de shōji 障子 (cloison coulissante tendue de papier translucide), mais reliant en douceur (fuwari to ふわりと) jardin et zashiki 座敷 (pièce de réception, salon). Cet espace ambivalent, ou cette bordure d'espaces (kükan no fuchi 空間の縁), est vraiment contraire au principe du tiers exclu. C'est grâce à lui justement que tant la pureté du zashiki que la simplicité du jardin peuvent mutuellement se mettre en valeur et s'épanouir ensemble. Cette bordure de 
lieu (basho no fuchi 場所の縁) est véritablement un calage (eji 依止) conjuguant par leur en 縁 deux espaces connexes ${ }^{3}$.

Dans le vocabulaire paysager, beaucoup de mots incarnent cette relation : piémont, rive, etc. Si yama no ha 山の端 (épaulement, contrefort) indique la ligne de crête d'une montagne, yamagiwa 山際 (bord de la montagne) est la forme d'un ciel clair bordé par la montagne. C'est par l'éclairage mutuel du ciel et de la montagne que l'un et l'autre existent. Le paysage naît de cette nouaison d'un en entre ciel et montagne.

\subsection{La composition co-suscitative (okiawase 置き合わせ)}

L'arrangement des tobiishi 飛び石 (pierres-à-sauter, pas japonais) ou des plantations dans un jardin, la terminologie utilisée pour la composition (okiawase 置き合わせ) des éléments plastiques dans l'ikebana 生花 ou d'autres arts traditionnels, comme les notions triangulaires de shin 真 (cœur), soe 添え(annexe), hikae 控え(adjoint), etc., enseignent à bien saisir le ma dans la liaison des éléments entre eux, pour les embellir tout en tirant parti des imperfections ou des irrégularités que leurs formes tiennent de la nature. Les fleurs ou les pierres étant vides ( $k \bar{u}$ 空) de nature propre (jishō 自性) d'elles-mêmes, elles renaissent plus belles quand on les combine et les compose à d'autres éléments. La beauté des choses n'est pas une réalité en soi (sokujiteki jitsuzai 即自的害在), elle fleurit dans les fluctuations de leur entrelien. Cette esthétique médiale (füdoronteki na bigaku 風土論的な美学) est un processus génératif extrêmement mouvant et dynamique.

3. Engawa 縁側 et ma 間. Littéralement, engawa est le "bord " d'un espace. L'engawa adjacent au salon est une véranda planchéiée, normalement non couverte de tatamis, avec un plafond bas et sobre qui s'étend comme un auvent vers le jardin et auquel peut s'adjoindre un petit engawa exposé à la pluie, nure-en 濡孔縁. L'engawa est donc un espace intermédiaire entre l'extérieur et l'intérieur. Tous ces éléments, jardin, espace sous-auvent, engawa et salon constituent une série formant le bel endroit dans la maison japonaise authentique, produisant une gradation de l'ombre très nuancée. C'est ainsi que la personne assise dans le salon en face du jardin est invitée à nouer un lien co-suscitatif (en) avec la nature grâce a cet intervalle vide à gradation ambiguë et nuancée $(m a)$. L'engawa peut s'appeler simplement en 縁, nouant le lien co-suscitatif entre l'homme et la nature. 
Le moi n'existe pas en lui-même, il s'instaure à la lumière de son environnement, et ce caractère dépendant est valable dans la relation co-suscitative entre les montagnes et les maisons, les fleuves et les arbres qui constituent le paysage. En somme, qu'il s'agisse du moi, des montagnes, des fleuves ou des arbres, c'est dans le champ et l'état de leur composition mutuelle que s'éclaire leur existence respective. Ce haïku de Buson 囬村 en est témoin :

$\begin{array}{lll}\text { 五月雨や } & \text { Samidare ya } & \text { Ah pluie de mousson } \\ \text { 大河を前に } & \text { taiga o mae ni } & \text { devant le grand fleuve } \\ \text { 家二軒 } & \text { ie niken } & \text { deux maisons }\end{array}$

Ces deux maisons de rang qui commencent à dialoguer, leur composition avec la rive du grand fleuve est devenue paysage.

\subsection{Paysage d'emprunt (shakkei 借景) et voir-comme (mitate 見立て)}

Cette pensée de la dépendance paysagère, pour laquelle les divers phénomènes n'existent pas en soi mais de par l'arrangement, la composition, les reflets où leur forme se manifeste, l'on peut dire que c'est une pensée médiale (füdoteki 風土的), une pensée de la co-suscitation dans l'éclairage mutuel, où les choses dépendent du champ ( $b a$ 場). C'est exactement de la même façon que l'emprunt de paysage $\left(s h a k k e i^{4}\right)$, comme fracassant la structure géographique, relie de façon contingente la montagne A et le jardin $\mathrm{B}$, initialement sans rapport entre eux, et de même que le procédé du

4. Shakkei : paysage d'emprunt. Art jardinier pour capturer vivantes et introduire dans le jardin d'avant-plan des montagnes ou d'autres scènes assez loin au delà de l'enceinte privée. Celles-ci sont prises dans le cadre composé par les lignes horizontales de la haie, de l'auvent ou de l'engawa, et par les lignes verticales des arbres ou des piliers. La montagne et le jardin, comme indiqué dans le texte, se relient de manière contingente, produisant un sens nouveau grâce à la liaison co-suscitative qui en résulte. Les éléments naturels lointains, ainsi invités dans le jardin et le salon, tendent à perdre leur distance et leur objectivité. Il deviennent des éléments culturels. Le temple Entsūji 円通寺 à Kyoto en présente un modèle typique. 
voir-comme (mitate $\left.e^{5}\right)$, génère un sens nouveau par la liaison co-suscitative (en 緑) qui en résulte.

L'emprunt de paysage est pour ainsi dire une citation sémiologique insérée dans le contexte qu'est le jardin. De tels exemples se retrouvent dans le bouddhisme et le shintoïsme avec la pratique du kanjo 钓請 (“invitation ", par exemple inviter un Bouddha ou un bodhisattva à venir dans l'aire rituelle lors d'une cérémonie). Souvent, c'est une cérémonie religieuse pour consacrer un temple reproduisant, dans une province éloignée, un temple original, bouddhiste ou shintoïste, situé près d'une montagne ou d'un fleuve sacrés. L'on trouve par exemple dans tout le Japon de nombreux rejetons du Sengen jinja 浅間神社, où le culte est rendu au mont Fuji, ou du Kumano hongū 熊野本宮. Dans de nombreux cas, ils sont installés près de basses collines boisées qui, par "voir-comme », sont appelées « montagne ».

Par exemple, à Tokyo, les bords du plateau diluviale de Musashino (Musashino daichi 武蔵野台地), au contact avec la plaine alluviale, abondent en coteaux et vallées de dix à vingt mètres de dénivelée. Beaucoup de temples bouddhistes ou shintoïstes s'y sont localisés, les voyant comme des montagnes. Ces coutumes interprètent le champ terrestre en termes géomantiques (chiso 地相 ${ }^{6}$ ), lui conférant un sens particulier ; méthode où l'on peut voir un aspect du « voir-comme».

5. Jardin de promenade (kaiyūshiki teien 回遊式庭園), parcours circulaire (kaiyu 回 遊), mitate. Contrairement au jardin destiné à être regardé d'un point de vue fixe à partir du salon, le jardin de promenade est conçu de telle façon qu'on puisse s'amuser des paysages successifs et dramatiques au fur et a mesure que se déplace le promeneur. Ce type de jardin s'est développé largement à l'époque d'Edo dans les domaines des seigneurs féodaux. Le Kōrakuen 後楽園 et le Hamarikyū 浜離宮, à Tokyo, sont des cas représentatifs.

L'aménagement de ce type de jardins s'est inspiré de la tradition de visite des hauts lieux ou lieux renommés (meisho 名所) par les poètes pèlerins. Un jardin de promenade est conçu comme une miniature du territoire, dotée de plusieurs meisho. Dans ce contexte, les éléments paysagers introduits symboliquement sont appelés mitate 見立て (regarder-comme). Il convient également de se rappeler la mentalité ésotérique qui s'est manifestée typiquement dans le parcours de pèlerinage reliant 88 temples de Shikoku, où l'on exécute ses dévotions corporelles sur la terre sacrée.

6. Chisō. Phase physionomique du champ terrestre, concept typiquement développé surtout en Chine continentale et en Corée comme manière géomantique d'augurer d'un site de résidence ou de tombe. Dans ce but, plusieurs patrons géomorphologiques composés de montagne et de rivière ont été développés. Cette méthode de lecture du 


\section{Lieu et limite sacrée (kekkai 結界)}

Marquer un lieu par un $k e k k a i^{7}$ est un usage consistant à le délimiter, physiquement et symboliquement, par un ma entre le dedans et le dehors. Il s'agit par exemple d'un shimenawa (torsade de paille sacrée) enroulé autour d'un grand arbre visité par les dieux, autour d'un «siège de roc " (iwaza 磐 座, rocher où descendent les dieux), ou, autre exemple, du portique (torii 鳥居) marquant l'entrée d'un temple shintoïste. Ces marquages viennent d'une discrimination symbolique entre le monde profane et une aire sacrée. Ils se sont ensuite répandus pour délimiter l'intérieur et l'extérieur de façon générale, telles les murailles d'un château, les palissades ou les haies entourant une résidence, puis les habitations populaires. Ils donnent ainsi de la profondeur à l'espace en mettant en scène les bordures de champ ( $b a 0$ fuchidoru enshutsu 場を縁取る演出). Par une opposition différentielle (shisateki tairitsu 指差的対立) qui sémantise mutuellement le profane et le sacré, la limite entre intérieur et extérieur acquiert du sens, nouaison dont les termes n'ont en eux-mêmes aucune substance.

Comme le montre bien le mot même de kekkai, il y a là en même temps liaison ( $k e t s u$ 結) et délimitation ( $k a i$ 界). Il ne s'agit pas de la construction d'une substance, mais d'un dispositif éphémère, qui se noue et se dénoue au gré des circonstances. Cela révèle par excellence l'émanation d'un

champ terrestre et son sens, beaucoup moins rigoureux et doctrinaires, mais plus esthétiques et paysagers, ont longuement subsisté au Japon, où l'on a l'habitude de donner autant d'importance à l'environnement terrestre qu'à la composition architecturale des bâtiments. C'est ainsi que le champ terrestre est devenu un élément culturel et patrimonial, en particulier dans le cas des meisho (hauts lieux). À Edo, le chisō avec la montagne sacrée du Fuji-san au dos donnant sur l'eau au sud de la baie d'Edo, a été extrêmement admiré par les poètes ainsi que par les citadins. En tant que lieu, chisō garde très fidèlement son caractère de champ terrestre.

7. Kekkai. Originellement, des objets de culte tels qu'arbres, rochers ou autres éléments naturels pouvaient être symboliquement sanctifiés et proclamés kekkai par des shimenawa 注連縄 (cordes de paille avec papiers décoratifs). Plus tard, les haies, portes, ruisseaux, etc. définissant ou entourant l'enceinte des temples shintö̈stes et bouddhistes, ont été assimilés à des kekkai. Aujourd'hui, ce mot s'applique plus largement aux haies ou portes de toutes sortes entourant un domaine résidentiel profane. Différenciant l'espace intérieur de l'extérieur, le kekkai en tant que dispositif reflétant un certain sens du champ est aussi important que le sont les façades en Occident. 
être-lié (kechienteki sonzai 結緑的存在) insubstantiel, où la co-dépendance du dedans et du dehors qualifie l'espace.

Le kekkai, à la lettre, «lie », ou «noue » (musubu 結ぶ) la limite (kai 界). Refusant les formations immobilières, laissant présager une prochaine dissolution, son simple appareil relève d'une esthétique de la transience (utsuroi 移ろい). Selon les besoins, le kekkai se diversifie. Ce peut être, comme le sudare 簾 (store de jonc ou de bambou), un rideau laissant filtrer la vue de l'intérieur, ou la coupant comme le hei 塀 (mur d'enceinte); mais même dans le second cas, le dépassement des frondaisons du jardin qui est là-derrière laisse planer une ambiance où le dedans et le dehors sont vaguement liés tout en restant distincts. En un mot, la démarcation éphémère du kekkai met en scène la différence même de deux espaces, le dedans et le dehors.

\section{Les parcours internes du lieu}

Dans l'impression que l'on a d'un terrain quelconque, les creux et les bosses dessinent des contours fragmentaires, qui se distribuent en choses et que la perception subjective articule en groupements d'objets localisés. Cependant, plutôt que par la vue, qui est intentionnelle et puissamment objectifiante, l'étendue terrestre qu'est la situation, par les sensations tactiles de notre corps qui sont inintentionnelles, nous fait intuitivement percevoir des champs plutôt que des choses. Cette atmosphère impalpable (kasukana kehai 幽かな気配) parcourt, comme un faible courant électrique, le corps qui s’y déplace.

Une excursion (kaiyu 回遊 $\left.^{8}\right)$, c'est l'action de se plonger dans un lieu, et en détruisant la structure géographique des montagnes, des rivières, des chemins, de les transformer en circonstances formées au gré du temps, pour se baigner dans l'atmosphère au champ généreux des sensations corporelles qui en jaillissent.

Par exemple, quand on regarde les Trente-six vues du mont Fuji (Fugaku sanjūrokkei 富嶽三十六景 de Katsushika Hokusai 暮飾北斎, au fil du déplacement du point de vue, les premiers plans tous singuliers du paysage se succèdent les uns après les autres, capturant chaque fois le mont Fuji, tout

8. Voir note 5. 
petit au loin. Celui-ci est objectifié par la netteté de sa silhouette, qui se détache par le dehors, mais son piémont, qui se fond dans le lieu, n'est plus un objet vu du dehors : il est entraîné à l'intérieur du lieu par la chorégraphie des corps, et s'y engloutit. Le frottement des corps en excursion intériorise le lieu.

Faire la tournée des sanctuaires bouddhiques est une coutume répandue, par exemple les quatre-vingt-huit étapes de Shikoku (Shikoku hachi-jūhachi henro 四国八十八遍路), les trente-trois étapes de Kannon dans les provinces de l'ouest (Saigoku sanjūsan sho 西国三十三所) et de l'est (Bandō sanjūsan kasho 坂東三十三筒所), le tour des sept dieux de la fortune (Shichi fukujin meguri 七福神巡り) ou celui des huit paysages (Hakkei meguri 八 景巡り), etc. Tous ces circuits demandent de parcourir la terre, et dissolvent ainsi l'expérience du champ dans le temps. Ce faisant, ils démembrent les perceptions visuelles, qui cherchent à saisir le monde en tant qu'objet làdehors, et ils nous invitent, en quelque sorte, à "voir le monde de l'intérieur ». On ne peut pas ne pas penser au rituel populaire du «tour de la matrice en Kannon " (Kannon no tainai meguri 観音の胎内巡り). Il y faut entrer dans une caverne aménagée dans une grande statue de Kannon, y chercher son chemin en tâtonnant dans l'obscurité de cette caverne, assimilée à la matrice de Kannon, pour en ressortir à la clarté. On fait par là fictivement l'expérience de la vie et de la mort, et l'on prie pour le bonheur de son corps purifié. Là, il n'y a qu'une intériorité poussée à l'extrême, empêchant le moi d'objectiver le monde par le dehors. Ce faisant, l'être humain ne fait-il pas l'expérience d'une intégration fictive de son corps au cosmos, et de la continuité de la vie et de la mort ?

\section{Articulation sémiotique et sociabilité}

Le mot niwa (niha) 庭, où l'on voit le sens premier de $b a$ 場, signifie aujourd'hui le jardin d'une résidence entourée par un mur, mais il semble qu'à l'origine il désignait un espace où la communauté accomplissait rites et tâches en commun, fêtes, travaux agricoles ou marchés. Les jardins seigneuriaux de l'époque d'Edo sont le type même de cette utilisation du jardin comme lieu où se tenaient de pareilles manifestations. Le jardin était la scène où le beau monde se jouait, sous la forme de festins. On y échangeait 
des poèmes, on s'y adonnait aux rites corporels de la boisson, tout en jouissant du paysage.

Suivant d'anciennes coutumes, les hauts lieux paysagers du territoire Matsushima 松島, Ama-no-hashidate 天橋立, le mont Fuji, etc. - étaient représentés en miniature dans les jardins-promenades (kaiyüshiki teien 回遊式庭園 ${ }^{9}$ ), le toponyme remédiant aux imperfections matérielles du substitut. Les toponymes ne servent pas seulement à mémoriser et à utiliser les lieux, ils leur donnent sens. Ainsi Matsu-no-chaya 松の茶屋 (la Buvetteaux-pins), Shimizu-no-chaya 清水の茶屋 (la Buvette-aux-eaux-pures), Tsutsuji-ga-oka 榴ヶ岡 (la Butte-aux-azalées), Fujimi-yama 富士見山 (le mont Mire-Fuji), Tsukimidai 月見台 (le plan Mirelune), Shiomi-yama 汐見 山 (le mont Miramar), Shio-hama 汐浜 (la Plage-aux-marées), etc.

Ces lieux étaient nommés en fonction des particularités du paysage qu'ils offraient à la vue, ou des formes de sociabilité qui s'y tenaient. Dans les jardins, les changements de propriétaire s'accompagnaient souvent d'une révision complète de la toponymie. Les fréquentes promenades que l'on y faisait étaient l'occasion de concours de poésie, où tout en faisant le tour du jardin, l'on jouait sur les thèmes proposés par le propriétaire. Les jardins ne font pas seulement fleurir les fleurs, mais aussi les mots. Tels étaient les jardins japonais.

Bien entendu, les jardins ne sont pas seuls en cause. Une infinité de toponymes parsèment l'espace géographique : noms de montagnes, de routes, de villes, de ponts... Les lieux habités ne sont pas seulement articulés par la vue et par les sens du corps, ils le sont également par les signes linguistiques, qui leur confèrent un sens.

Ainsi, d'innombrables espèces de mots sont semés dans les lieux, permettant au moi isolé de devenir un " moi social " (aidagara no watakushi 間柄の私), tandis que le paysage observé par le moi (watakushi no keikan 私の景観) devient un paysage vécu par le nous (wareware no fükei 我々の 風景). Les liens sociaux invisibles s’incarnent dans le paysage que tissent les mots échangés et les manières partagées, autrement dit dans la vie du corps social. Cette incarnation est fort analogue à celle des liens familiaux par la maison, qui en est la construction visible.

9. Voir note 5 . 
Semés dans l'espace, les mots lient les humains aux lieux par le sens qui en germe, et aussi les humains entre eux. L'on peut ainsi dire que, dans le champ ainsi ensemencé, les mots lèvent comme signes médiateurs de co-suscitation (kechien baikai kigo 結縁媒介記号) entre lieux et humains, et humains entre eux. De cette union des lieux et des mots procède la tradition lettrée de l'image-poème (shiga itchi 詩画一致). Le poète peint une demeure idéale au sein du paysage. Ses amis lettrés y calligraphient un éloge (shisan 詩讃) de la vie raffinée qu’il y mène.

\section{Vacillation et complexité du champ}

Emboîtements et échappées de la vue, bassins fluviaux, relief, variété du couvert végétal... Dans ce phénomène culturel qu' est la jouissance du paysage, avec toute la complexité et la superposition de ses lieux divers, le vent, la lumière, le cycle des saisons, ces manifestations de la vie et de sa mouvance animent le paysage, elles lui donnent vie.

Dans la chaleur étouffante de l'été, au crépuscule, le frais tintement de la clochette-à-vent (fürin 風鈴), sous l'avancée du toit, est un poème à la fraîche (nōryō no fübutsu-shi 納涼の風物詩), secourant les humains accablés par l'été nippon. Par la grâce de la clochette, un imperceptible courant d'air déclenche dans l'atmosphère un frémissement du champ, se transforme en la fraîcheur d'une sonorité, se répercute et s'amplifie. C'est un exemple de ces motifs mésologiques ( fübutsu 風物) que systématisent les recueils de mots saisonniers (saijiki 歳時記), et qui sont la règle dans l'aire culturelle nippone.

Accompagnant le passage des saisons et le clair-obscur de l'écoulement des jours (chūya no kageroi 昼夜のかげろい), germe, respire, croît puis disparait le goût de la nature (füryū 風流, littéralement, " à la manière ou à l'image du vent»), celui de la neige, de la lune et des fleurs d'une esthétique du champ (ba no bigaku場の美学). Constituant la base du sens esthétique et de l'aménité au Japon, cela donne une tournure naturelle à un style de vie élégant, éco-symbolique, dans toutes les facettes de la sociabilité.

Là, tout attachement est banni, et révérée au contraire l'incessante expression du renouveau dans la négation. C'est que véritablement, comme le disait Bashō, "les changements du ciel et de la terre sont la graine de l'élégance" (kenkon no hen wa füga no tane 乾坤の変は風雅の種). Ce que 
voulait Bashō, c'est puiser l'atmosphère du champ où le plongeait la fluctuation des circonstances, dans sa mouvance fluidifiant le dur contour des substances.

\section{Conclusion}

1. Le paysage n'est pas un objet opposé au sujet ; il symbolise la nouaison de l'entrelien d'un lieu, dans sa mouvante complexité, avec un corps en vie.

2. Le $m a$, champ corporel où se déploient des rapports de co-suscitation, rend compte des principaux phénomènes paysagers.

3. Émanant de l'acausalité co-suscitative des choses, le paysage naît d'un processus historique.

4. Entre lieu, corps et langage, à la fois onde (mouvance) et particule (sémiose), le paysage est l'insubstantiel et fluctuant aspect d'un milieu humain (fuyu suru füdoteki kūsōo 浮遊する風土的空相).

Traduit par Augustin Berque

\section{Références}

BARIDON Michel, 1998

Les Jardins, Paris, Robert Laffont.

BERQUE Augustin, 1986

Le Sauvage et l'Artifice. Les Japonais

devant la nature, Paris, Gallimard.

\section{GIBSON James, 1979}

The Ecological Approach to Visual

Perception, Boston, Houghton Mifflin.

LYNCH Kevin, 1963

The Image of the City, Cambridge, MA, MIT Press. 
MIYOSHI Manabu 三好学, 1905-1914

Nihon shokubutsu keikan 日本植物景観 (Les paysages végétaux du Japon),

Tokyo, Maruzen 丸善.

\section{DŌGEN 道元, 1990}

Shōbōgenzō 正法眼蔵 (Trésor de l'CFil de la Vraie Loi), annoté par MIZUNO Yaoko 水野弥穗子, t. 1, Chap. III : « Genjō kōan » 現成公案, Tokyo, Iwanami shoten 岩波書店.

\section{TSUJIMURA Tarō 辻村太郎, 1937}

Keikan chirigaku kōwa 景観地理学講話

(Cours de géographie du paysage),

Tokyo, Chijin shokan 地人書館.

\section{UEXKÜLL Jakob von}

\section{\& Georg KRISZAT, 1934}

Streifzüge durch die Umwelten von Tieren und Menschen (Incursions dans les mondes ambiants animaux et humains), Berlin/Frankfurt am Main, Fischer Verlag.

YAMANOUCHI Tokuryū 山内徳立, 1974 Rogosu to Renma ロゴスとレンマ (Logos et Lemme), Tokyo, Iwanami shoten 岩波書店. 